\title{
Coherent synchrotron radiation by electrons moving on circular orbits
}

\author{
Yunhai Cai \\ SLAC National Accelerator Laboratory, 2575 Sand Hill Road, Menlo Park, California 94205, USA
}

(Received 2 January 2017; published 14 June 2017)

\begin{abstract}
We study coherent synchrotron radiation by electrons in the Frenet-Serret coordinate system with a constant curvature $1 / \rho$. Based on the Hamiltonian in the Courant-Synder theory of particle accelerators, we find in general that the transverse force is essentially the Lorentz force but with a substitution of the transverse magnetic field $B_{x, y} \rightarrow(1+x / \rho) B_{x, y}$, where $x$ and $y$ are the transverse positions. The curvature term provides us a key to derive the point-charge wakefield explicitly in terms of the incomplete elliptic integrals of the first and second kind, resulting in a steady-state theory of the coherent synchrotron radiation in two-dimensional free space.
\end{abstract}

DOI: 10.1103/PhysRevAccelBeams.20.064402

\section{INTRODUCTION}

Coherent synchrotron radiation (CSR) in the bending magnets is one of the most important limits to the advance of the brightness of the electron beam in storage rings for infrared and terahertz radiation [1-3] and bunch compressors [4] in free-electron-laser (FEL) light sources [5] and high-energy linear colliders. In rings, it induces microwave instability [6,7], causing bursting [8] of synchrotron radiation. In compressors, it increases the energy spread and causes microbunching $[9,10]$. An advance in the understanding of CSR has played an important role in the design and operation of the FEL facilities and can have a great impact for future accelerators.

Historically, the one-dimensional theory of CSR was developed in terms of a simple longitudinal wakefield [11-14]. These wakes were implemented in the code Elegant [15], which is widely used in simulations for the design of magnetic bunch compressors. The simulations are largely in good agreement with the experimental observations [4]. However, as a theory itself, it is not quite complete because it simply ignores the transverse force without any explanation. Our goal in this paper is to develop a twodimensional theory and lay a solid foundation for further advances toward a complete theory of CSR.

The importance of the transverse force of CSR was first emphasized by Talman [16] and later by Carlsten and Raubenheimer [17]. The study was followed with the calculation of the transverse wake by Derbenev and Shiltsev [18] and later refined by Stupakov [19]. There were similar studies in simulations [20-23]. However, the theory is never fully developed largely because of subtleties

Published by the American Physical Society under the terms of the Creative Commons Attribution 4.0 International license. Further distribution of this work must maintain attribution to the author(s) and the published article's title, journal citation, and DOI. in dealing with curvature, relativistic effect, and singularity. In this paper, we will continue their efforts and start with the curvature.

\section{EQUATION OF MOTION}

Given the scalar and vector potentials,

$$
\begin{gathered}
\vec{E}=-\nabla \phi-\frac{1}{c} \frac{\partial \vec{A}}{\partial t}, \\
\vec{B}=\nabla \times \vec{A}
\end{gathered}
$$

in the curvilinear coordinate system shown in Fig. 1, the Hamiltonian is well known [24],

$$
\mathcal{H}_{s}\left(x, p_{x}, y, p_{y}, t,-H ; s\right)=-\left(1+\frac{x}{\rho}\right) \mathcal{P}_{s}-\frac{e}{c} \mathcal{A}_{s},
$$

where

$$
\begin{aligned}
& \mathcal{P}_{s}=\sqrt{\left(\frac{H-e \phi}{c}\right)^{2}-\left(p_{x}-\frac{e}{c} A_{x}\right)^{2}-\left(p_{y}-\frac{e}{c} A_{y}\right)^{2}-m^{2} c^{2}} \\
& \mathcal{A}_{s}=\left(1+\frac{x}{\rho}\right) A_{s} .
\end{aligned}
$$

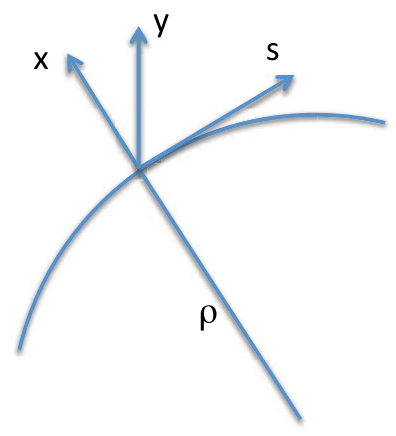

FIG. 1. The curvilinear coordinate system. 
Three Hamilton equations lead to

$$
\begin{aligned}
& \dot{x}=\frac{\partial \mathcal{H}_{s}}{\partial p_{x}}=\left(1+\frac{x}{\rho}\right) \frac{p_{x}-\frac{e}{c} A_{x}}{\mathcal{P}_{s}}, \\
& \dot{y}=\frac{\partial \mathcal{H}_{s}}{\partial p_{y}}=\left(1+\frac{x}{\rho}\right) \frac{p_{y}-\frac{e}{c} A_{y}}{\mathcal{P}_{s}}, \\
& \dot{t}=\frac{\partial \mathcal{H}_{s}}{\partial(-H)}=\left(1+\frac{x}{\rho}\right) \frac{H-e \phi}{c^{2} \mathcal{P}_{s}} .
\end{aligned}
$$

Using Eqs. (5) along with the Hamillton equations, $\dot{p}_{x}=-\partial \mathcal{H}_{s} / \partial x, \dot{p}_{y}=-\partial \mathcal{H}_{s} / \partial y$, we derive the equations

$$
\begin{array}{r}
\left(\frac{\hat{x} \mathcal{P}_{s}}{1+\frac{x}{\rho}}\right)^{\prime}=\frac{\mathcal{P}_{s}}{\rho}+\frac{e}{c}\left\{c \hat{t} E_{x}+\left[\hat{y} B_{s}-\left(1+\frac{x}{\rho}\right) B_{y}\right]\right\}, \\
\left(\frac{\hat{y} \mathcal{P}_{s}}{1+\frac{x}{\rho}}\right)^{\prime}=\frac{e}{c}\left\{c \hat{t} E_{y}+\left[\left(1+\frac{x}{\rho}\right) B_{x}-\dot{x} B_{s}\right]\right\}
\end{array}
$$

that govern the transverse motion. For a uniform magnetic field $B_{0}$ in the vertical direction, Eq. (6) reduces to

$$
\left(\frac{x p_{s}}{1+\frac{x}{\rho}}\right)^{\prime}=\frac{p_{s}}{\rho}-\frac{e}{c}\left(1+\frac{x}{\rho}\right) B_{0},
$$

where $p_{s}=\sqrt{p^{2}-p_{x}^{2}-p_{y}^{2}}$ and $p$ is the momentum of the particle. Applying Eq. (8) to the design particle with momentum $p_{0}$, we find that the field has to satisfy

$$
B_{0}=\frac{c p_{0}}{e \rho}
$$

Substituting it back into Eq. (8), we have

$$
\left(\frac{\dot{x} p_{s}}{1+\frac{x}{\rho}}\right)^{\prime}=\frac{p_{s}-p_{0}}{\rho}-\frac{x p_{0}}{\rho^{2}}
$$

Using the paraxial approximation $p_{s} \approx p$ and ignoring the nonlinear terms, the equation of the horizontal motion becomes

$$
x^{\prime \prime}+\frac{x}{\rho^{2}}=\frac{\delta}{\rho},
$$

where $\delta=\left(p-p_{0}\right) / p_{0}$.

If there are additional electromagnetic fields, including self-fields, present, we can substitute the expression $B_{0}$ in Eq. (9) into Eq. (6) as an extra field and obtain the equation of the horizontal motion

$$
\begin{aligned}
& \left(\frac{\dot{x} \mathcal{P}_{s}}{1+\frac{x}{\rho}}\right)^{\prime}+\frac{x p_{0}}{\rho^{2}} \\
& \quad=\frac{\mathcal{P}_{s}-p_{0}}{\rho}+\frac{e}{c}\left\{c \hat{t} E_{x}+\left[y B_{s}-\left(1+\frac{x}{\rho}\right) B_{y}\right]\right\} .
\end{aligned}
$$

Now, the bending field $B_{0}$ is excluded explicitly.

Using again the paraxial approximation and ignoring the nonlinear terms similar to the previous case, we simplify Eqs. (12) and (7) to

$$
\begin{aligned}
x^{\prime \prime}+\frac{x}{\rho^{2}} & =\frac{\delta}{\rho}+\frac{e}{c p_{0} \beta_{s}}\left[E_{x}+\beta_{y} B_{s}-\beta_{s}\left(1+\frac{x}{\rho}\right) B_{y}\right], \\
y^{\prime \prime} & =\frac{e}{c p_{0} \beta_{s}}\left[E_{y}+\beta_{s}\left(1+\frac{x}{\rho}\right) B_{x}-\beta_{x} B_{s}\right],
\end{aligned}
$$

where $\beta_{x}, \beta_{y}$, and $\beta_{s}$ are the components of $\vec{\beta}=\vec{v} / c$, the velocity normalized to the speed of light. The transverse motion is driven by the electric and magnetic fields. The transverse force is the expected Lorentz force but with extra terms that are proportional to $x / \rho$. The importance of these terms will become clear later.

For the energy change, it is easier to start with $d E / d t=$ $e \vec{v} \cdot \vec{E}$ and then we have

$$
\delta=\frac{e}{c p_{0} \beta_{s} \beta} \vec{\beta} \cdot \vec{E}
$$

\section{ELECTRIC AND MAGNETIC FIELDS}

Now we consider the relativistic effect in computing the fields. For simplicity, we assume that all particles are moving in the horizontal plane. In fact, this simplification is consistent with the 2D theory of CSR since the particles will remain in the plane as we will see later. When a charged particle is moving on a circular orbit from point $\mathrm{A}$ to $\mathrm{B}$ as illustrated in Fig. 2, it generates electromagnetic fields, which can be expressed by the Lienard-Wiechert formula [25],

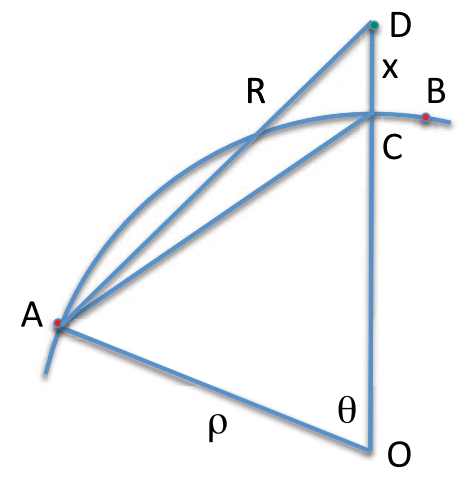

FIG. 2. A source particle (at A) moves along a circular orbit and radiates electromagnetic fields that reach a testing particle (at D). 


$$
\vec{E}=e\left[\frac{\hat{n}-\vec{\beta}}{\gamma^{2}(1-\vec{\beta} \cdot \hat{n})^{3} R^{2}}\right]_{\mathrm{ret}}+\frac{e}{c}\left[\frac{\hat{n} \times\{(\hat{n}-\vec{\beta})\} \times \dot{\vec{\beta}}}{(1-\vec{\beta} \cdot \hat{n})^{3} R}\right]_{\mathrm{ret}},
$$

$$
\vec{B}=\hat{n} \times \vec{E},
$$

where $\hat{n}=\vec{R} / R$ and $\vec{R}$ is the vector from the point A, where the source particle is at the retarded time $t^{\prime}$, to D the observation point at the time $t$. The retarded time is given by

$$
t^{\prime}=t-\frac{R}{c} \text {. }
$$

The first term in Eq. (15) is due to space charge and is not important for ultrarelativistic electrons because of the suppression of a factor of $1 / \gamma^{2}$. In this paper, we ignore the space-charge term and focus only on the radiative term as the source of the coherent synchrotron radiation. This approach is recently introduced [26] to analyze the longitudinal effect in a 2D model.

The acceleration is defined as $\dot{\vec{\beta}}=d \vec{\beta}\left(t^{\prime}\right) / d t^{\prime}$. With the radius $\rho$ of the circle, we have $\dot{\beta}=c \beta^{2} / \rho$. Using the acceleration and trigonometry, it is straightforward to compute the electromagnetic fields. Their nonvanishing components can be written as

$$
\begin{gathered}
E_{s}=\frac{e \beta^{2}[\cos 2 \alpha-(1+\chi)][(1+\chi) \sin 2 \alpha-\beta \kappa]}{\rho^{2}[\kappa-\beta(1+\chi) \sin 2 \alpha]^{3}}, \\
E_{x}=\frac{e \beta^{2} \sin 2 \alpha[(1+\chi) \sin 2 \alpha-\beta \kappa]}{\rho^{2}[\kappa-\beta(1+\chi) \sin 2 \alpha]^{3}}, \\
B_{y}=\frac{e \beta^{2} \kappa[(1+\chi) \sin 2 \alpha-\beta \kappa]}{\rho^{2}[\kappa-\beta(1+\chi) \sin 2 \alpha]^{3}},
\end{gathered}
$$

where $\alpha=\theta / 2$ and $\kappa=R / \rho$ is given explicitly,

$$
\kappa=\sqrt{\chi^{2}+4(1+\chi) \sin ^{2} \alpha},
$$

with $\chi=x / \rho$. There are many equivalent expressions of the fields given the relation in Eq. (21). Here, we have chosen the ones with simple factors, especially in the denominator. Otherwise, the numerical noise will overwhelm the fields near the singularity.

\section{RETARDED CONDITION}

Since the expressions of the fields are subject to the retarded condition in Eq. (17), we need to solve the condition in terms of the position of the source particle at the moment $t$. During the time interval $t-t^{\prime}$, the source particle moves from A to $\mathrm{B}$ along the arc with speed $v$ as illustrated in Fig. 2. So the arc length between point $\mathrm{C}$ and $\mathrm{B}$ is given by

$$
\ell=v\left(t-t^{\prime}\right)-\left(s-s^{\prime}\right),
$$

where $s-s^{\prime}$ is the arc length between A and C. Moreover, if we introduce $z=s-v t$ as the longitudinal position, then Eq. (22) leads to $\ell=z^{\prime}-z$. Using the retarded condition in Eq. (17), we can rewrite Eq. (22) in terms of $\alpha$,

$$
\xi=\alpha-\frac{\beta}{2} \sqrt{\chi^{2}+4(1+\chi) \sin ^{2} \alpha},
$$

where $\xi=-\ell / 2 \rho$. Given $\xi$ and $\chi$, the angle $\alpha$ can be found with a Newton search using Eq. (23).

Expanding up to the fourth order of $\alpha$, Eq. (23) can be rewritten as

$$
\begin{aligned}
\alpha^{4} & +\frac{3\left(1-\beta^{2}-\beta^{2} \chi\right)}{\beta^{2}(1+\chi)} \alpha^{2}-\frac{6 \xi}{\beta^{2}(1+\chi)} \alpha \\
+ & \frac{3\left(4 \xi^{2}-\beta^{2} \chi^{2}\right)}{4 \beta^{2}(1+\chi)}=0 .
\end{aligned}
$$

The solution of this quartic equation is given in the Appendix. A comparison between the numerical and analytical solutions is carried out. The difference $\gamma \Delta \alpha$ is at a level of $10^{-6}$ in the plotting region in Figs. 3 and 4. The agreement is so good that we use the analytic solution for its computational efficiency in this paper.

\section{FORCES}

Knowing $\alpha$ and reading from Eqs. (13) and Eqs. (18)-(20), the transverse force is given by

$F_{x}=\frac{e^{2} \beta^{2}[\sin 2 \alpha-\beta(1+\chi) \kappa][(1+\chi) \sin 2 \alpha-\beta \kappa]}{\rho^{2}[\kappa-\beta(1+\chi) \sin 2 \alpha]^{3}}$

and $F_{y}=0$ so that the particles stay in the horizontal plane.

Using $\alpha$, we compute and plot $E_{s}$ and $F_{x}$ in Fig. 3. The scalings of the plots are chosen according to their approximated invariance with respect to $\gamma$. Note that $F_{x} \sim \gamma^{3}$ instead of $\gamma^{4}$ in the vicinity of the origin because of the cancellation between the electric and magnetic forces. The singularity of the transverse force is clearly seen in the right plot. Because of these complicated features when two particles are approaching each other, it is often difficult to use $E_{s}$ and $F_{x}$ as Green's functions for a bunch distribution in practice.

It is worth noting that in the asymptotic region where $\mu=3 \gamma^{3} \xi \gg 1$ and $\chi=0$, our results of $E_{s}=$ $2 e /\left[\rho^{2}(6 \xi)^{4 / 3}\right]$ and $F_{x}=e^{2} /\left[\rho^{2}(6 \xi)\right]$ fully agree with the known results in the $1 \mathrm{D}$ theory $[12,27]$.

\section{POTENTIALS}

Here we continue on dealing with the singularity. A method to avoid the singularity in the Green's functions is integration by parts. It requires us to know the integral of the Green's function. Differentiating the retarded condition in Eq. (23), we have 

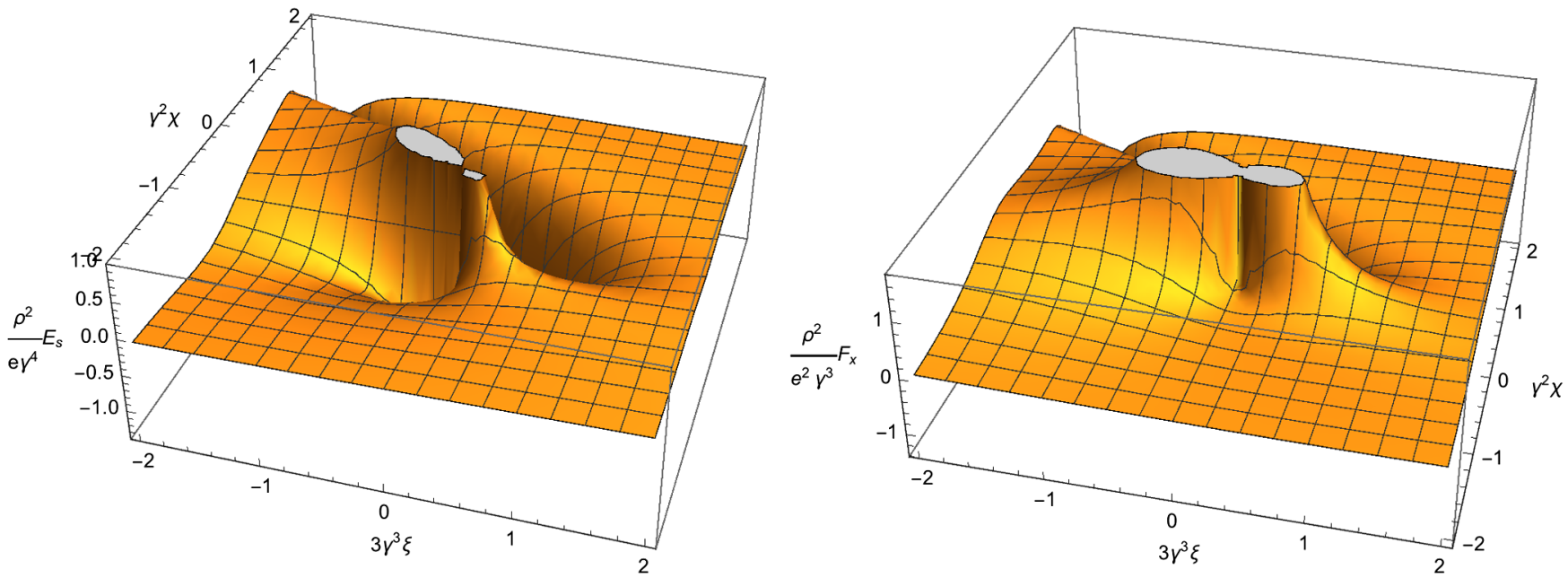

FIG. 3. The scaled longitudinal field $E_{s} \rho^{2} / e \gamma^{4}$ (left) and transverse force $F_{x} \rho^{2} / e^{2} \gamma^{3}$ (right) as a function of $\mu=3 \gamma^{3} \xi$ and $\nu=\gamma^{2} \chi$ with $\gamma=500$.
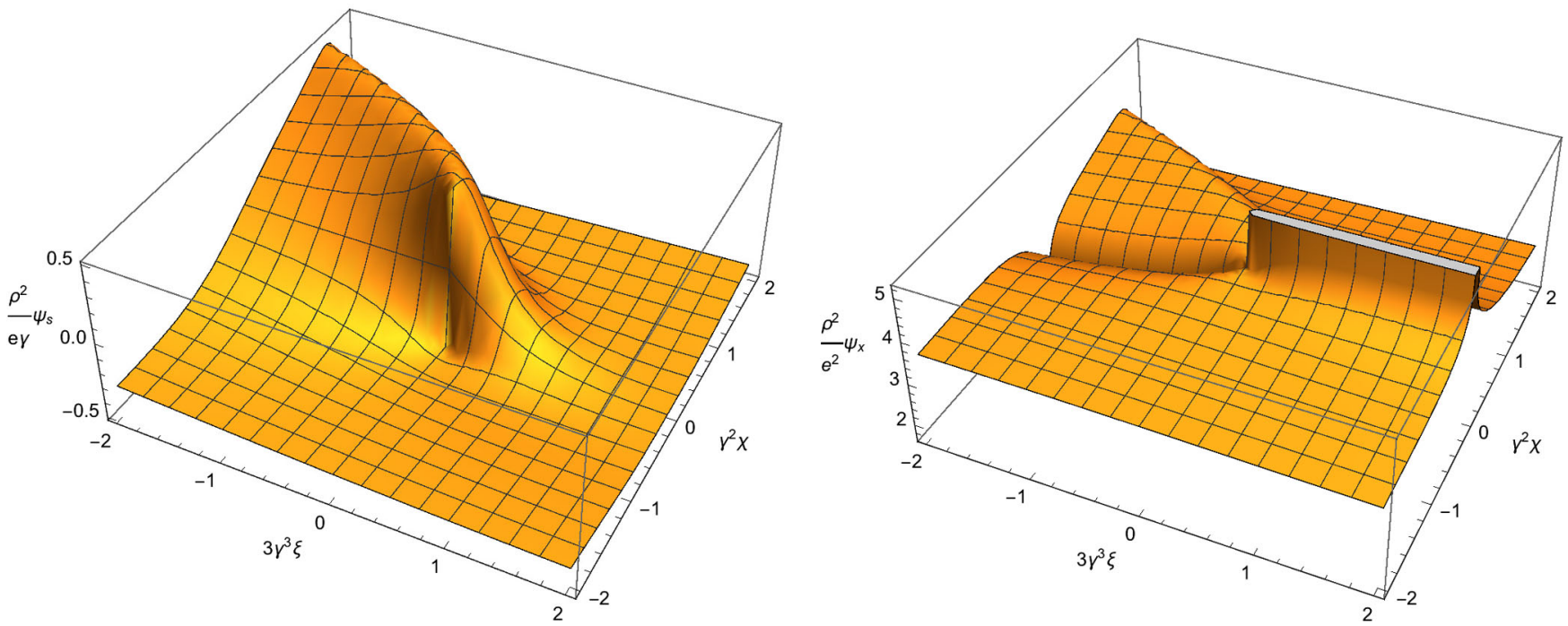

FIG. 4. The scaled longitudinal potential $\psi_{s} \rho^{2} / e \gamma$ (left) and transverse potential $\psi_{x} \rho^{2} / e^{2}$ as a function of $\mu=3 \gamma^{3} \xi$ and $\nu=\gamma^{2} \chi$ with $\gamma=500$.

$$
d \xi=\left(1-\frac{\beta(1+\chi) \sin 2 \alpha}{\kappa}\right) d \alpha .
$$

Combining it with the longitudinal electric field $E_{s}$ in Eq. (18), we find

$$
\begin{aligned}
E_{s} d \xi & =\frac{e \beta^{2}[\cos 2 \alpha-(1+\chi)][(1+\chi) \sin 2 \alpha-\beta \kappa]}{\rho^{2} \kappa[\kappa-\beta(1+\chi) \sin 2 \alpha]^{2}} d \alpha \\
& =d\left\{\frac{e \beta^{2}\left(\cos 2 \alpha-\frac{1}{1+\chi}\right)}{2 \rho^{2}[\kappa-\beta(1+\chi) \sin 2 \alpha]}\right\} .
\end{aligned}
$$

This relation naturally leads to a longitudinal potential,

$$
\psi_{s}(\xi, \chi)=\frac{e \beta^{2}\left(\cos 2 \alpha-\frac{1}{1+\chi}\right)}{2 \rho^{2}[\kappa-\beta(1+\chi) \sin 2 \alpha]},
$$

noting that the dependence of $\xi$ is through $\alpha(\xi, \chi)$ obtained as the solution of the retarded condition and $E_{s}=d \psi_{s} / d \xi$.

Similarly, for the transverse force, we have $F_{x}=d \psi_{x} / d \xi$ with a transverse potential,

$$
\begin{aligned}
\psi_{x}(\xi, \chi) & \\
= & \frac{e^{2} \beta^{2}}{2 \rho^{2}}\left\{\frac { 1 } { | \chi | ( 1 + \chi ) } \left[\left(2+2 \chi+\chi^{2}\right) F\left(\alpha, \frac{-4(1+\chi)}{\chi^{2}}\right)\right.\right. \\
& \left.-\chi^{2} E\left(\alpha, \frac{-4(1+\chi)}{\chi^{2}}\right)\right] \\
& +\frac{\kappa^{2}-2 \beta^{2}(1+\chi)^{2}+\beta^{2}(1+\chi)\left(2+2 \chi+\chi^{2}\right) \cos 2 \alpha}{\beta(1+\chi)\left[\kappa^{2}-\beta^{2}(1+\chi)^{2} \sin ^{2} 2 \alpha\right]} \\
& \left.-\frac{\kappa\left[1-\beta^{2}(1+\chi) \cos 2 \alpha\right] \sin 2 \alpha}{\left[\kappa^{2}-\beta^{2}(1+\chi)^{2} \sin ^{2} 2 \alpha\right]}\right\},
\end{aligned}
$$



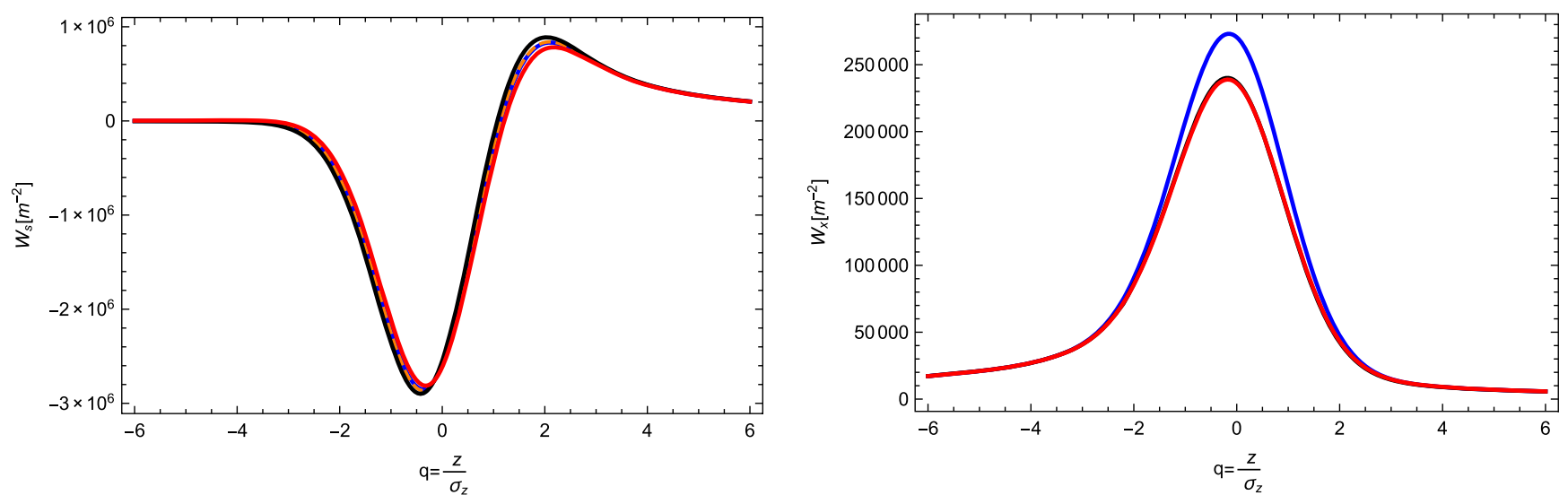

FIG. 5. The longitudinal (left) and transverse (right) wakes at various transverse positions $\chi=-\sigma_{\chi}$ (black), 0 (blue), and $\sigma_{\chi}$ (red) for a Gaussian bunch with $\sigma_{x}=\sigma_{z}=10 \mu \mathrm{m}$ on a curve with radius $\rho=1 \mathrm{~m}$ and $\gamma=500$.

where $F(\alpha, k)$ and $E(\alpha, k)$ are the incomplete elliptic integrals of the first and second kind, respectively. Note that the curvature term in the transverse force is necessary to obtain the expression.

The scaled potentials are plotted in Fig. 4. On the left, we can see that the potential $\psi_{s}$ is enhanced in the tangent direction when the source particle radiated at the retarded time. On the right, we see mostly the "logarithmic" singularity along the line of $\xi=0$ with a different sign in the region $\xi>0$ or $\xi<0$. Because of this singularity, the transverse force is infinite in the 1D theory. Fortunately, the integral over the 2D phase space is finite. This property allows us to compute the transverse wake for a smooth bunch distribution in the 2D theory. It is worth noting that the divergence comes from the term with the incomplete elliptic integral of the first kind.

\section{WAKE FIELDS}

Given the potentials in Eqs. (28) and (29), and the equations of the motion in Eqs. (13) and (14), we derive a change of the momentum deviation $\delta$ and a kick to $x$ by the bunch wakefields

$$
\begin{gathered}
\delta=\frac{r_{e} N_{b}}{\gamma} W_{s}(z, \chi), \\
x^{\prime \prime}=\frac{r_{e} N_{b}}{\gamma} W_{x}(z, \chi),
\end{gathered}
$$

where $r_{e}$ is the classical electron radius, $N_{b}$ the bunch population, and the wakes are

$$
\begin{aligned}
& W_{s}(z, \chi)=\iint \mathcal{Y}_{s}\left(\frac{z-z^{\prime}}{2 \rho}, \chi-\chi^{\prime}\right) \frac{\partial \lambda_{b}\left(z^{\prime}, \chi^{\prime}\right)}{\partial z^{\prime}} d z^{\prime} d \chi^{\prime}, \\
& W_{x}(z, \chi)=\iint \mathcal{Y}_{x}\left(\frac{z-z^{\prime}}{2 \rho}, \chi-\chi^{\prime}\right) \frac{\partial \lambda_{b}\left(z^{\prime}, \chi^{\prime}\right)}{\partial z^{\prime}} d z^{\prime} d \chi^{\prime},
\end{aligned}
$$

with $\mathcal{Y}_{s}=2 \rho \psi_{s} /\left(e \beta^{2}\right), \mathcal{Y}_{x}=2 \rho \psi_{x} /(e \beta)^{2}$, and $\lambda_{b}$ the distribution that is normalized to $\int \lambda_{b} d z d \chi=1$.
The wakes of a Gaussian bunch are shown in Fig. 5. The longitudinal wake is an order of magnitude larger than the transverse one. The deviation of the longitudinal wake from the 1D wake, plotted in dashed orange color in the left plot, is rather small. The transverse wake is mostly symmetric with respect to $\chi$ as shown in the right plot in Fig. 5. A nearly Gaussian profile can be approximated by

$$
W_{g}(q)=\frac{\Lambda}{\sqrt{2 \pi} \rho \sigma_{z}} \exp \left(-\frac{q^{2}}{2}\right),
$$

with the coefficient

$$
\Lambda=\ln \left[\frac{\left(\rho \sigma_{z}^{2}\right)^{2 / 3}}{\sigma_{x}^{2}}\left(1+\frac{\sigma_{x}}{\sigma_{z}}\right)\right] .
$$

This Gaussian wake is called the Talman force [18].

\section{EMITTANCE GROWTH}

In a short bending dipole of length $L_{B}$, the increase of normalized emittance due to the longitudinal wake is given by [27]

$$
\Delta \epsilon_{N}^{(s)} \approx 7.5 \times 10^{-3} \frac{\tilde{\beta}_{x}}{\gamma}\left(\frac{r_{e} N_{b} L_{B}^{2}}{\rho^{5 / 3} \sigma_{z}^{4 / 3}}\right)^{2},
$$

where $\tilde{\beta}_{x}$ is the beta function at the exit where the dispersion and its slope are assumed zero. The direct emittance growth due to the transverse wake can also be estimated using the Gaussian wake in Eq. (34) and written as

$$
\Delta \epsilon_{N}^{(x)} \approx \frac{(-3+2 \sqrt{3})}{24 \pi} \frac{\tilde{\beta}_{x}}{\gamma}\left(\frac{\Lambda r_{e} N_{b} L_{B}}{\rho \sigma_{z}}\right)^{2} .
$$

TABLE I. Parameters for the last bend in the second bunch compressor of Linac Coherent Light Source.

\begin{tabular}{lccccccc}
\hline \hline Parameter & $\gamma$ & $\epsilon_{N}$ & $\sigma_{z}$ & $N_{b}$ & $\tilde{\beta}_{x}$ & $\rho$ & $L_{B}$ \\
\hline Value & 10,000 & $0.5 \mu \mathrm{m}$ & $10 \mu \mathrm{m}$ & $10^{9}$ & $5 \mathrm{~m}$ & $5 \mathrm{~m}$ & $0.5 \mathrm{~m}$ \\
\hline \hline
\end{tabular}


For the parameters in Table I, the estimated emittance growth indirectly from the energy spread is $38 \%$ while the direct contribution from the transverse wake is $37.5 \%$. This example shows that the transverse force can significantly increase the beam emittance in the bunch compressors.

We have developed a steady-state theory of CSR in the $2 \mathrm{D}$ free space. The theory is self-consistent with a proper treatment of the singularity. It naturally approaches to the 1D theory at the limit of the transverse dimension going to zero. It is remarkable seeing how the geometry, relativity, and singularity have coherently played their own roles in the theory.

\section{ACKNOWLEDGMENTS}

I would like to thank Karl Bane, Kazuhito Ohmi, Gennady Stupakov, and Robert Warnock for many helpful discussions. Especially, I would like thank Yuantao Ding for providing the parameters of the bunch compressors in LCLS. This work was supported by the Department of Energy under Contract No. DE-AC02-76SF00515.

\section{APPENDIX: SOLUTION OF THE QUARTIC EQUATION}

In general, we would like to find the roots of the depressed quartic equation:

$$
\alpha^{4}+v \alpha^{2}+\eta \alpha+\zeta=0 .
$$

It has an analytical solution discovered by Ferrari. The method finds a value $m$ to factorize the fourth-order polynomial by a product of two quadratic polynomials. It turns out that $m$ itself is a solution of a third-order equation and is given by

$$
m=-\frac{v}{3}+\left(\frac{\zeta}{3}+\frac{v^{2}}{36}\right) \Omega^{-1 / 3}+\Omega^{1 / 3},
$$

where

$\Omega=\frac{\eta^{2}}{16}-\frac{\zeta v}{6}+\frac{v^{3}}{216}+\sqrt{\left(\frac{\eta^{2}}{16}-\frac{\zeta v}{6}+\frac{v^{3}}{216}\right)^{2}-\left(\frac{\zeta}{3}+\frac{v^{2}}{36}\right)^{3}}$.

Then we have

$\alpha=\left\{\begin{array}{ll}\frac{1}{2}\left(\sqrt{2 m}+\sqrt{-2(m+v)-\frac{2 \eta}{\sqrt{2 m}}}\right) & \text { if } \xi \geq 0 \\ \frac{1}{2}\left(-\sqrt{2 m}+\sqrt{-2(m+v)+\frac{2 \eta}{\sqrt{2 m}}}\right) & \text { if } \xi<0\end{array}\right.$.

These are two real roots of the quartic equation. They cover two different regions: ahead of or behind the source particle.
[1] M. Abo-Bakr, J. Feikes, K. Holldack, G. Wustefeld, and H.-W Hubers, Steady-State Far-Infrared Coherent Synchrotron Radiation detected at BESSY, Phys. Rev. Lett. 88, 254801 (2002).

[2] J. M. Byrd, W. P. Leemans, A. Loftsdottir, B. Marcelis, M. C. Martin, W. R. McKinney, F. Sannibale, T. Scarvie, and C. Steier, Observation of Broadband Self-Amplified Spontaneous Coherent Terahertz Synchrotron Radiation in a Storage Ring, Phys. Rev. Lett. 89, 224801 (2002).

[3] F. Sannibale et al., A Model Describing Stable Coherent Synchrotron Radiation in Storage Rings, Phys. Rev. Lett. 93, 094801 (2004).

[4] K. L.F. Bane et al., Measurements and modeling of coherent synchrotron radiation and its impact on the Linac Coherent Light Source electron beam, Phys. Rev. ST Accel. Beams 12, 030704 (2009).

[5] P. Emma et al., First lasing and operation of an ångstromwavelength free-electron laser, Nat. Photonics 4, 641 (2010).

[6] G. Stupakov and S. Heifets, Beam instability and microbunching due to coherent synchrotron radiation, Phys. Rev. ST Accel. Beams 5, 054402 (2002).

[7] K. L. F. Bane, Y. Cai, and G. Stupakov, Threshold studies of the microwave instability in electron storage rings, Phys. Rev. ST Accel. Beams 13, 104402 (2010).

[8] M. Venturini and R. Warnock, Bursts of Coherent Synchrotron Radiation in Electron Storage Rings: A Dynamical Model, Phys. Rev. Lett. 89, 224802 (2002).

[9] S. Heifets, G. Stupakov, and S. Krinsky, Coherent synchrotron radiation instability in a bunch compressor, Phys. Rev. ST Accel. Beams 5, 064401 (2002).

[10] Z. Huang and K.-J. Kim, Formulas for coherent synchrotron radiation microbunching in a bunch compressor chicane, Phys. Rev. ST Accel. Beams 5, 074401 (2002).

[11] Y. S. Derbenev, J. Rossbach, E. L. Saldin, and V. D. Shiltsev, DESY Print, TESLA-FEL 95-05, Hamburg, 1995.

[12] J. B. Murphy, S. Krinsky, and R. L. Gluckstern, Longitudinal wakefield for an electron moving on a circular orbit, Part. Accel. 57, 9 (1997).

[13] M. Dohlus and T. Limberg, Emittance growth due to wake fields on curved bunch trajectories, Nucl. Instrum. Methods Phys. Res., Sect. A 393, 494 (1997).

[14] E. L. Saldin, E. A. Schneidmiller, and M. V. Yurkov, On the coherent radiation of an electron bunch moving in an arc of a circle, Nucl. Instrum. Methods Phys. Res., Sect. A 398, 373 (1997).

[15] M. Borland, Simple method for particle tracking with coherent synchrotron radiation, Phys. Rev. ST Accel. Beams 2001) 070701,4$)$.

[16] Richard Talman, Novel Relativistic Effect Important in Accelerators, Phys. Rev. Lett. 56, 1429 (1986).

[17] B.E. Carlsten and T. O. Raubenheimer, Emittance growth of bunched beams in bends, Phys. Rev. E 51, 1453 (1995).

[18] Y. S. Derbenev and V. D. Shiltsev, Report No. SLAC-PUB7181, 1996.

[19] G. V. Stupakov, Proceedings of PAC97, Vancouver, British Columbia, Canada, Report No. SLAC-PEPRINT-2011034, 1997. 
[20] D. Gillingham and T. Antonsen, Calculation of coherent synchrotron radiation in toroidal waveguides by paraxial wave equation, Phys. Rev. ST Accel. Beams 10, 054402 (2007).

[21] R. Li, Curvature-induced bunch self-interaction for an energy-chirped bunch in magnetic bends, Phys. Rev. ST Accel. Beams 11, 024401 (2008).

[22] G. Bassi, J. Ellison, K. Heineman, and R. Warnock, Microbunching instability in a chicane: Two-dimensional mean field treatment, Phys. Rev. ST Accel. Beams 12, 080704 (2009).
[23] A. Novokhatski, Field dynamics of coherent synchrotron radiation using a direct numerical solution of Maxwell's equations, Phys. Rev. ST Accel. Beams 14, 060707 (2011).

[24] E. D. Courant and H. S. Snyder, Theory of the alternatinggradient synchrotron, Ann. Phys. (N.Y.) 3, 1 (1958).

[25] J. D. Jackson, Classical Electrodynamics, 3rd ed. (John Wiley \& Son, New York, 1999).

[26] C. Huang, T. J. T. Kwan, and B. Cartsten, Two dimensional model for coherent synchrotron radiation, Phys. Rev. ST Accel. Beams 16, 010701 (2013).

[27] G. V. Stupakov, Report No. SLAC-PUB-8028, 2006. 